\title{
PEDAGOGÍA VERDE PARA LA FORMACIÓN DE SERES CRÍTICOS Y REFLEXIVOS
}

\section{GREEN PEDAGOGY FOR THE FORMATION OF CRITICAL AND REFLECTIVE BEINGS}

\author{
Marco Vinicio Duque Romero ${ }^{1}$ \\ Karla Paola Cadena Bastidas ${ }^{2}$
}

Recibido: 2021-06-25 / Revisado: 2021-07-20 / Aceptado: 2021-08-12 / Publicado: 2021-09-15

Forma sugerida de citar: Duque-Romero, M. V. y Cadena-Bastidas, K. P. (2021). Pedagogía verde para la formación de seres críticos y reflexivos. Retos de la Ciencia. 5(e). 1-12. https://doi.org/10.53877/rc.5.e.20210915.01

\section{RESUMEN}

El avance de la ciencia y tecnología ha modificado el estilo de vida, implantando sitios de recreación superficiales que alejan del medio natural, la educación debe centrar al estudiante dentro del contexto natural para generar conciencia medioambiental y formar seres críticos y reflexivos. El objetivo del estudio es presentar alternativas que promuevan la interacción con el medio natural y desarrollar una conciencia críticareflexiva. El presente documento se sustentó en la investigación cualitativa por medio de la revisión bibliográfica, se seleccionaron los artículos más relevantes, los resultados se obtuvieron de revistas indexadas, principalmente en Google Scholar. La pedagogía verde pretende transformar el contexto social y cultural agregando a la naturaleza como eje central de la educación; para ello diversos investigadores presentan alternativas reales para acercar el entorno natural al social tratando de contrastar los daños causados al planeta. Varios investigadores tratan de generar conciencia medioambiental, analizar el contexto socio histórico y presentar alternativas que frenen los daños causas a la Tierra, para producir un cambio significativo dentro de la sociedad, para ello es necesario intervenir en la educación e ir moldeando las acciones, pensamientos y formas de proceder de los estudiantes de manera consiente, directa y real.

Palabras claves: educación ambiental, huertos escolares, pensamiento crítico, pedagogía verde, metodología TINI.

\footnotetext{
${ }^{1}$ Magister en Administración Educativa y Docencia Universitaria. Docente Investigador de la Universidad Tecnológica Indoamérica. Ecuador. E-mail: marcoduque@uti.edu.ec / ORCID: https://orcid.org/0000-0002-31980924

${ }^{2}$ Licenciada en Ciencias de Educación. Estudiante de Maestría en Innovación y Liderazgo Educativo en la Universidad Tecnológica Indoamérica. Ecuador. E-mail: kcadena@indoamerica.edu.ec / ORCID: http://orcid.org/0000-0001-7736-5057
} 


\section{ABSTRACT}

The advancement of science and technology has modified the lifestyle, implanting superficial recreation sites away from the natural environment, education must center the student within the natural context to generate environmental awareness and form critical and reflective beings. Present real alternatives that promote interaction with the natural environment and generate meaningful learning. This article was based on qualitative research through a bibliographic review, the most relevant articles were selected, the results were obtained from indexed journals, mainly in Google Scholar. Green pedagogy aims to transform the social and cultural context by adding nature as the central axis of education; For this, various researchers present real alternatives to bring the natural environment closer to the social one, trying to contrast the damage caused to the planet. Several researchers try to generate environmental awareness, analyze the socio-historical context and present alternatives that stop the damage caused to the Earth, to produce a significant change within society, for this it is necessary to intervene in education and to shape actions, thoughts and ways of proceeding of students in a conscious, direct and real way.

Keywords: environmental education, school gardens, critical thinking, green pedagogy, Tini methodology.

\section{INTRODUCCIÓN}

Para comenzar con el tema del presente estudio, es indispensable aludir al principio del tiempo, según Izaguirre (2014) manifiesta, el ser humano desde su inicio se ha inclinado a vivir en conjunto, sumando fuerzas y recursos entre sus semejantes para subsistir y desarrollarse; los individuos habitualmente interactuaban con la naturaleza, acoplándose a las condiciones que el planeta les imponía viviendo en una magnífica armonía con el medio natural; lastimosamente el transcurso del tiempo y modernización cambió a los seres humanos y su estilo de vida fue modificándose alejándolos de la naturaleza y de su contacto.

Esta investigación enfatiza el llamado del medio ambiente al cambio, pues los estudiantes necesitan una inyección de naturaleza, conciencia y respeto desde etapas tempranas hasta la etapa adulta siendo la educación herramienta fundamental para esta transformación (Díaz y Alemán, 2008). Es tiempo de enfatizar que el planeta Tierra presenta un riesgo latente debido a los diversos acontecimientos, daños locales y globales causados al medio ambiente: la contaminación, la destrucción de los recursos naturales, la sobrepoblación, la falta de conciencia y respeto por la naturaleza comprometen la salud de los ecosistemas y del planeta. Es preciso señalar que OMS - Organización Mundial de la Salud (2012) manifestó que la contaminación del aire fue responsable de 3.7 millones de muertes en el planeta [...]; alrededor del $88 \%$ de estas muertes ocurren en países de ingresos bajos y medios, más de 150 millones de personas en América Latina viven en ciudades que exceden las Guías de Calidad del Aire, emitidas por el mismo organismo en 1987.

Indiscutiblemente el oxígeno no sale del cemento, cuando se entienda esto se sabrá la importancia de la educación ambiental. Desde el punto de vista de Limón et. al. (2002) citados en Cabrera (2014), "el cambio comienza con los principios de la Educación Ambiental, pues esta tiene como objetivos formar y crear conciencia sobre entorno natural y social [...], formando hombres y mujeres como individuos comprometidos, solidarios y responsables con el medio ambiente" (p. 2). 
Cierto es que los conocimientos aportan al futuro de la sociedad, tanto como las acciones, los comportamientos, los hábitos y las posturas de los seres humanos destruyen o sostienen al medio ambiente. Educar para un mundo mejor sí es posible, la educación de los niños es el pilar de nuestra sociedad (Díaz y Alemán, 2008); para ello es necesario educarlos bajo una cultura medioambiental constructivista. Desde el punto de vista de otros autores en investigaciones del tema, declaran que:

La esencia de la vida en comunidad se basa en la posibilidad de elucidar e integrar los mejores rasgos de los individuos que la constituyen, es necesario que la educación, como instrumento de socialización y de actitud crítica, adopte respuestas válidas para los retos que tiene planteados la humanidad. (Novo, 2009)

La Pedagogía Verde es un conjunto de conceptos, ideas y estrategias para acompañar el desarrollo humano cultivando el vínculo de amor hacia la tierra y todos los seres que la habitan (Freire, 2018); es fundamental para los seres humanos, pues el contacto con la naturaleza permite la maduración el sistema nervioso y del organismo desde las primeras etapas de la infancia, además permite construir una identidad autónoma, en relación de interdependencia con los demás seres vivos, genera actividad física, desarrolla las capacidades psicomotoras y la inteligencia espacial, mejora la salud en general y genera una conciencia crítica y reflexiva. Pérez y Belletich (2018) consideran que la Pedagogía Verde construye la naturaleza interna de los niños, y su necesidad de crecer en cada una de sus dimensiones humanas: física, emocional, social, intelectual y espiritual.

El enfoque educativo aborda cuestiones profundas que ponen en relación los principios de la pedagogía activa con el medio natural, Díaz y Alemán (2008) señalan que la educación posee tres funciones: la primera es la preservadora, garantiza la continuidad y cohesión; la segunda, el desarrollo permite formar personas críticas y creativas para generar conocimientos, da respuesta desde un enfoque histórico cultural a los problemas actuales y futuros; y la tercera es la promoción, que refuerza a las dos anteriores, prepara a los estudiantes para difundir los nuevos conocimientos que se van presentando con el transcurso del tiempo. Las instituciones educativas están a cargo de una gran responsabilidad, pues se pretende liderar una transformación cultural sin precedentes, que permita evolucionar desde una sociedad biofóbica que llena de miedo, perjuicios que no actúa de manera frontal con la Naturaleza, y la destruye, hacia una sociedad biofílica (de bios=vida y filos=amor), considerando que eje primordial es el amor y el cuidado a la vida. Se ha verificado que la Naturaleza es el mejor medio para un desarrollo y un aprendizaje holístico (del griego hólos=todo, que implica todas las dimensiones del ser humano) y saludable. (Freire, 2018)

El entorno natural debería ser el conductor del aprendizaje, fusionando los contenidos que trata el currículo. Freire (2018) denota que "la naturaleza es madre y es maestra" (p. 4), de esta concepción se puede fomentar una cultura de amor por la tierra, adoptando una perspectiva no-antropocéntrica que permita aprender a convivir, de manera práctica aumentando las relaciones y la frecuencia de contacto, emprendiendo un proceso de transformación aptitudinal generando un proyecto educativo biométrico.

La pedagogía verde enmarcará las bases pedagógicas de la educación actual, es fundamental que las áreas de estudio se enriquezcan y se hagan más significativas, con el tratamiento de temas que corresponden a los ejes transversales que son instrumentos básicos y valiosos para la mejor comprensión, análisis y transformación de la realidad. Para comenzar con la aplicación de esta, se requiere que las instituciones educativas abran sus puertas a la naturaleza, sacando la educación del 
aula o a la vez articular los tiempos y combinando la convivencia en espacios interiores y exteriores, ambos son complementarios en el proceso de aprendizaje. El vínculo con la Naturaleza va mucho más allá de explicar, señalar carteles, observar documentales, analizar fotografías y paisajes; es una manera de vivir, de sentir, de ser y de relacionarse con los demás. Freire (2011) establece que el contacto con la naturaleza es vital para el desarrollo cognitivo y psicomotriz ya que reduce los niveles de estrés y ansiedad que la escolaridad produce, favorece las funciones cognitivas (memoria, atención, concentración); por tanto, la presente investigación tiene como objetivo presentar alternativas reales que promuevan la interacción con el medio natural y desarrollar una conciencia crítica-reflexiva.

\section{METODOLOGÍA}

Este estudio es cualitativo, ya que, se basa en la recolección de información altamente confiable; Patton (1980) citado en Anguera (1986) asegura que la finalidad de este tipo de etudios es orientar al lector dentro del tema guiándolo al contexto, sin incluir percepciones, dado que pueden liar deducciones y convertirlas en suposiciones.

Además, el estudio se realizó por medio de la revisión bibliográfica, a través de la revisión y análisis crítico de la información para establecer relaciones y comparaciones entre las fuentes teóricas, garantizando su validez y relevancia (Gómez-Luna et. al., 2014). Para este estudio se contó con información de libros, artículos científicos, tesis doctorales, entrevistas en periódicos, entre otros, empleando recursos electrónicos como Google Scholar para garantizar la veracidad y confiabilidad de los datos, cuadros, citas, tablas y argumentos propuestos.

En el proceso se seleccionaron inicialmente veinte y cinco fuentes de revisión bibliográfica, que posteriormente se filtraron acorde a la calidad del contenido y método expuesto. Entre los recursos que apoyan y sustentan esta investigación se encuentran: Proyecto Verde, Huertos escolares; Proyecto TINI, Ministerio de Educación del Ecuador; Carta a la Tierra; Pensamiento Crítico; y, Datos estadísticos de la Organización Mundial de la Salud. En la búsqueda de fuentes por medios electrónicos se utilizaron las siguientes palabras claves: "Pensamiento crítico", "Pedagogía Verde" "huertos escolares", "Educación ambiental", "Metodología TINI"; además se consideraron fuentes actualizadas, priorizando artículos publicados en revistas científicas en el período de tiempo 2000-2021, realizando contrastes para descartar algunos y seleccionar los más idóneos para la investigación.

\section{RESULTADOS}

La pedagogía Verde pretende una transformación social y cultural que convierta la naturaleza en el eje primordial de la educación, Freire (2020) citado en Fresneda (2018) señala "Antes que salvar el planeta, vamos a amar el planeta" (p. 1), el conocimiento brota del contacto con el medio ambiente a la par con la ética del cuidado, libertad, solidaridad y democracia; a continuación, se presenta en Figura 1 alternativas que abordan la Pedagogía Verde. 
Figura 1:

Pedagogía Verde

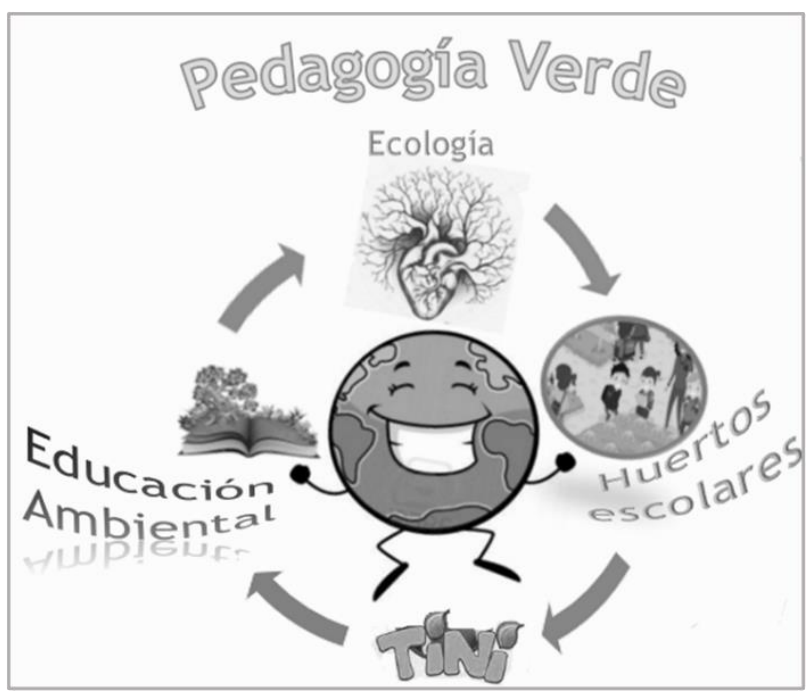

Elaborado por: Elaboración propia

La Figura 1 indica alternativas que apoyan al desarrollo de la pedagogía verde que contribuyen a preservar, amar, respetar y cuidar el medio ambiente, además desempeñan un papel importante dentro de la sociedad para fomentar el desarrollo sostenible del ambiente. Los aportes de cada alternativa se detallan a continuación:

La Metodología TINI (Tierra de Niños, Niñas y adolescentes), que según manifiesta su autor, Joaquín Leguía en una entrevista para El Periódico del Campo. (El Productor, 2017):

Consiste en un área que le entregan a niños, niñas y jóvenes, que puede ser desde medio metro de tierra hasta o inclusive una maceta con tres plantas, puede ser un bosque, en cualquier área en diferentes ecosistemas, donde ellos desarrollen acciones que cuiden la vida y la biodiversidad, que los beneficien a ellos mismos, a la biodiversidad y a la naturaleza.

El contacto con la naturaleza genera múltiples beneficios en el desarrollo cognitivo, físico, social y emocional de los miembros de la comunidad educativa, pues facilita al docente el desarrollo de competencias en todas las áreas curriculares; además, promueve el pensamiento sistémico al ver cómo estas se articulan para un propósito en común (Mieles y Vergara, 2020). Desde el punto de vista de Novo (2009):

La educación se enfrenta, cuando menos, a dos retos ineludibles: por un lado, el reto ecológico, que implica contribuir a formar y capacitar no sólo a jóvenes y niños, sino también a los gestores [...]; por otro, el desafío social que, en un mundo en el que la riqueza está muy injustamente repartida, nos impele a transformar radicalmente las estructuras de gestión y redistribución de los recursos de la Tierra.

La Educación Ambiental desempeña un rol importante tanto en la educación como en la sociedad, cuidar la naturaleza es cuidar la vida, así se garantiza una mejor calidad de vida para las actuales y futuras generaciones (Cabrera, 2014). El valor del cuidado de los seres que habitan el planeta, la exploración de la convivencia entre todos los individuos y lo que posibilita la vida, evocan frescura y amplitud de miradas; Gomera (2008), afirma que "los conocimientos, percepciones, conductas y actitudes son dimensiones que, en conjunto, conforman el concepto de conciencia, esta contribuye a la formación integral de la persona y a su educación en todos los niveles" (p. 2), paralelamente a los autores mencionados. Pasek (2004) concibe a la 
Educación Ambiental como una nueva visión para sustituir y revisar las concepciones humanas en relación con el ambiente.

Algo semejante ocurre con el desarrollo de la ecología que, según Bermúdez y Longhi (2008) aseguran, genera profundos aprendizajes abarcando el contexto natural con el formal, pues posee enfoques que engloban los contenidos del currículum; mientras que Gonzáles (2002) concibe que la ecología trata de mantener nexos para generar una conciencia medioambiental mediante los ejes transversales que posee la educación.

Los Huertos escolares según manifiesta Medina (2020), son "laboratorios naturales que poseen vida, consiste en un terreno de pequeñas proporciones, por lo general cercado, en el que se prepara la tierra para la siembra de plantas, verduras, legumbres, árboles frutales, entre otros" (p. 1); estos brindan una oportunidad de acercar la naturaleza a las instituciones educativas, permiten a los estudiantes construir sus propios aprendizajes y conocimientos desde sus primeros años de formación académica; además desarrollan hábitos, costumbres, respeto, valores y conciencia hacia el medio ambiente. Como señala Castillo (2021), La educación conlleva a la socialización del ser, basada en normas, valores y principios (p. 2).

Se afirma que el huerto escolar es un recurso pedagógico que interrelaciona las diferentes áreas curriculares y favorece el desarrollo de las competencias básicas (Botella et. al., 2014). Cabe denotar que los huertos escolares permiten la interacción de todos los miembros de la comunidad educativa, convirtiendo a los estudiantes en los protagonistas del proceso apoyados por los docentes y los padres de familia, además contribuyen a edificar una cultura de paz, trabajo cooperativo, responsabilidad y respeto; García (2003) afirma que los Huertos Escolares son terapéuticos, pues reducen significativamente los niveles de estrés y ansiedad que la escolaridad produce. Existen varios autores que expone aportes encaminados a la Pedagogía Verde, los cuales se presentan en la Tabla 1.

Tabla 1:

Resumen de los principales aportes de fuentes bibliográficas

Autor Aporte

Gonzáles (2002) La conducta ecológica se explica por los valores, creencias y comportamientos individuales y colectivos con relación al medio ambiente.

García (2003) Los huertos escolares son la fuente para aproximar la naturaleza a los estudiantes, genera experiencias palpables.

Pasek (2004) La Educación Ambiental pretende que los estudiantes entiendan cómo se producen los fenómenos naturales y las causas de los factores sociales que inciden en su normal funcionamiento.

Gomera (2008) Las dimensiones cognitiva, afectiva, conativa y activa forman directrices hacia la conciencia ambiental, la educación pretende alcanzar el desarrollo de esta para incorporar al individuo de manera intencional.

Bermúdez y La Educación Ambiental posee enfoques que enriquecen los ejes del

Longhi (2008) currículum a manera de alcanzar profundos aprendizajes acerca del medio natural.

Novo (2009) Educar apoya al progreso de la sociedad, encamina hacia a la renovación de la calidad de vida y progreso hacia la convivencia, reedifica los principios del valor de la naturaleza, y contribuye a la equidad social y la diversidad cultural. 


\begin{tabular}{|c|c|}
\hline Cabrera (2014) & $\begin{array}{l}\text { La Educación Ambiental busca que la sociedad adopte actitudes, } \\
\text { conciencia, acciones y conductas razonables que determinen y } \\
\text { comprendan la problemática ambiental, reseña socio histórico y } \\
\text { estado actual del planeta. }\end{array}$ \\
\hline Izaguirre (2014) & $\begin{array}{l}\text { El avance de la ciencia y tecnología ha modificado el estilo de vida, la } \\
\text { sociedad ha implantado sitios de recreación superficiales que alejan } \\
\text { del contacto natural. }\end{array}$ \\
\hline $\begin{array}{l}\text { Botella, } \\
(2014)\end{array}$ & $\begin{array}{l}\text { El huerto escolar integra las diversas áreas curriculares, facilita el } \\
\text { desarrollo de competencias elementales. }\end{array}$ \\
\hline $\begin{array}{l}\text { Leguía y } \\
\text { Paredes (2016) }\end{array}$ & $\begin{array}{l}\text { La metodología TINI, su propósito es fomentar y consolidar una } \\
\text { cultura y conciencia medioambiental en las instituciones educativas. }\end{array}$ \\
\hline $\begin{array}{l}\text { Mieles y Vergara } \\
(2020)\end{array}$ & $\begin{array}{l}\text { Una cultura proactiva se afianza por medio de la educación, } \\
\text { compromete a los individuos con el medio natural. }\end{array}$ \\
\hline
\end{tabular}

Elaborado por: Elaboración propia

Gonzáles (2002), explica la estrecha relación entre el ser humano y la naturaleza, reflexionando que la vida humana depende de las condiciones del planeta, por lo que se busca medidas que detengan los daños causados al mismo. García (2003) expone que la mayor parte de la población habita en zonas urbanas alejadas del contexto natural creando brechas y provocando nostalgia al campo; los huertos escolares devuelven el sentir a las actividades agrícolas primordiales para la vida y cultura de la sociedad. Pasek (2004), manifiesta que la educación ambiental permite al docente abordar las problemáticas existentes y reflexionar acercar de las mismas para llegar a comprender el llamado de la naturaleza; mientras que Gomera (2008) afirma que la educación ambiental pretende lograr respeto e influir con las decisiones de la persona y resolver los problemas ambientales. Según Bermúdez y Longhi (2008), los estudiantes adoptarán comportamientos adecuados en base a los conocimientos impartidos. Para Cabrera (2014), la Educación Ambiental busca que la sociedad adopte actitudes, conciencia, acciones y conductas razonables que determinen y comprendan la problemática ambiental, reseña socio histórico y estado actual del planeta. Mientras que Izaguirre (2014), afirma que la educación debe centrar al niño dentro del contexto natural y generar un equilibrio entre la realidad actual. Por su parte, Botella et. al. (2014), indican que los huertos escolares brindan la oportunidad a los estudiantes de conocer su contexto, generar experiencias y relacionarlas con los diversos ejes transversales para trabajar en grupo, resolver situaciones inesperadas y potenciar la iniciativa individual. Leguía y Paredes (2016), expone que por medio de (la Metodología) TINI se pretende la integración y transversalización del enfoque medioambiental, para generar individuos socialmente responsables; en acuerdo con esta metodología. Mieles y Vergara (2020), adoptan una postura similar, argumenta que (la Metodología) TINI conduce a la aplicación de los conocimientos en el entorno para fortalecer y lograr los objetivos de una educación de calidad.

Son necesarias orientaciones que contribuyan a reconectar la experiencia, a redescubrir la naturaleza y a reencontrar la vida; varios autores manifiestan diversos aportes ante las alternativas, por ello es necesario categorizarlos para tener una visión más amplia; la Tabla 2 permite observar qué autores mantienen posturas similares. 
Tabla 2:

Categorización de Propuestas por Aportes

\begin{tabular}{|c|c|c|c|c|c|c|c|c|c|c|}
\hline Aspectos / Autores & 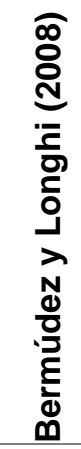 & 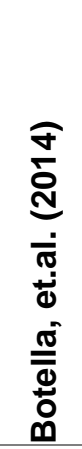 & 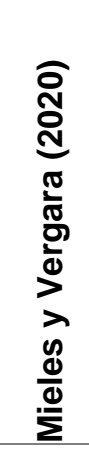 & 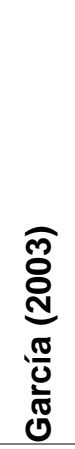 & 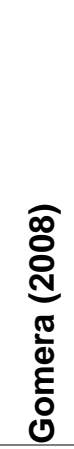 & 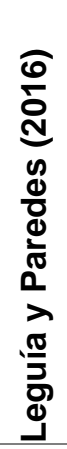 & 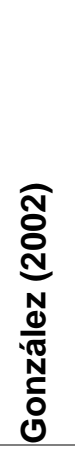 & 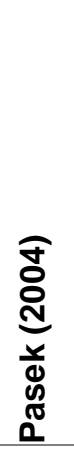 & 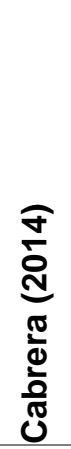 & \\
\hline \multicolumn{11}{|l|}{ Categorías } \\
\hline Huertos Escolares & & $X$ & & $X$ & & & & & & 2 \\
\hline Metodología Tini & & & $\mathrm{X}$ & & & K & & & & 2 \\
\hline Educación Ambiental & & & & & $\mathrm{X}$ & & & $\mathrm{X}$ & $X$ & 3 \\
\hline Desarrollo Ecológico & $\mathrm{X}$ & & & & & & $X$ & & & 2 \\
\hline
\end{tabular}

Elaborado por: Elaboración propia

Se puede reflexionar que un buen número de aportes coinciden en la educación ambiental, sin embargo, en las alternativas como huertos escolares, metodología TINI y Desarrollo ecológico mantienen posturas similares entre sí; además se puede apreciar que los primeros investigadores interesados en el crear una conciencia ambiental crítica-reflexiva son Gonzáles (2002) y García (2003); también esta temática se sigue abordando dentro de los estudios publicados entre los últimos 5 años.

Como hallazgos en las Tablas 1 y 2 se identificaron autores con posturas similares, por tanto, la Tabla 3 presenta una síntesis que abarca los aportes de los distintos autores que apoyan al desarrollo de la pedagogía verde:

Tabla 3:

Categorización de Propuestas por Aportes

\begin{tabular}{|c|c|c|}
\hline Categoría & Autor & Síntesis \\
\hline \multirow{2}{*}{$\begin{array}{l}\text { Huertos } \\
\text { Escolares }\end{array}$} & Botella, et.al. (2014) & \multirow{2}{*}{$\begin{array}{l}\text { Generan contacto con la naturaleza e integran las } \\
\text { diversas áreas curriculares y competencias que } \\
\text { demanda la educación. }\end{array}$} \\
\hline & García (2003) & \\
\hline \multirow[b]{2}{*}{$\begin{array}{l}\text { Metodología } \\
\text { TiNi }\end{array}$} & 2016) & \multirow{2}{*}{$\begin{array}{l}\text { Cultura proactiva que conduce a fortalecer y lograr } \\
\text { los objetivos de la educación, mediante la } \\
\text { integración y transversalización del enfoque } \\
\text { medioambiental. }\end{array}$} \\
\hline & Mieles y Vergara (2020) & \\
\hline \multirow{3}{*}{$\begin{array}{l}\text { Educación } \\
\text { Ambiental }\end{array}$} & Cabrera (2014) & \multirow{3}{*}{$\begin{array}{l}\text { Abarca las dimensiones cognitiva, afectiva, conativa } \\
\text { y activa que forman directrices hacia la conciencia } \\
\text { ambiental de manera intencional en base al respeto, } \\
\text { para generar bases sólidas para el desarrollo } \\
\text { sostenible del medioambiente. }\end{array}$} \\
\hline & Pase & \\
\hline & Gomera (2008) & \\
\hline \multirow[b]{2}{*}{$\begin{array}{l}\text { Desarrollo } \\
\text { Ecológico }\end{array}$} & Bermúdez y Longhi (2008) & \multirow{2}{*}{$\begin{array}{l}\text { Valores, creencias y comportamientos individuales y } \\
\text { colectivos, que permiten alcanzar profundos } \\
\text { aprendizajes en base a los conocimientos impartidos } \\
\text { para el pleno desarrollo del medio ambiente. }\end{array}$} \\
\hline & Gor & \\
\hline
\end{tabular}

Elaborado por: Elaboración propia 
Se ha encontrado dentro de la Tabla 3 que tres de seis autores se inclinan a la alternativa de la educación ambiental, mientras que entre pares sostienen a los huertos escolares, metodología TINI y desarrollo ecológico como alternativas para el desarrollo de una conciencia ambiental crítica-reflexiva, dejando a criterio personal la aplicación de estas.

El presente estudio ha reunido información altamente confiable y veraz para su redacción y sustento, es por ellos que a continuación se presenta la Tabla 4; en la que se expone el método, tipo de estudio y técnicas empleadas para la recolección de la información.

Tabla 4:

Métodos y Materiales

\begin{tabular}{|c|c|c|c|c|c|c|c|c|c|}
\hline \multirow[b]{2}{*}{ Autor(es) - año } & \multirow[b]{2}{*}{$\begin{array}{l}\text { Método } \\
\text { utilizado }\end{array}$} & \multirow[b]{2}{*}{$\begin{array}{l}\text { Tipo de } \\
\text { estudio }\end{array}$} & \multicolumn{5}{|c|}{$\begin{array}{l}\text { Técnica utilizada } \\
\text { para la recolección } \\
\text { de información }\end{array}$} & \multicolumn{2}{|c|}{$\begin{array}{l}\text { Sustento } \\
\text { teórico } \\
\text { del } \\
\text { método }\end{array}$} \\
\hline & & & $\begin{array}{l}\frac{\pi}{d} \\
\frac{d}{\partial} \\
\frac{0}{W}\end{array}$ & 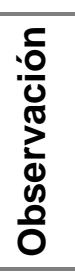 & 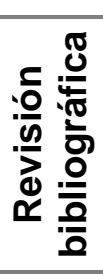 & 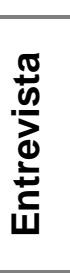 & $\begin{array}{l}\text { o } \\
\text { ơ }\end{array}$ & Sí & No \\
\hline $\begin{array}{c}\text { Botella, et. al. } \\
(2014)\end{array}$ & Investigativo & $\begin{array}{l}\text { Cuantitativo } \\
\text { y cualitativo }\end{array}$ & $X$ & & $x$ & $X$ & & & $X$ \\
\hline Cabrera (2014) & Cuantitativo & Descriptivo & $X$ & & & & & & $x$ \\
\hline $\begin{array}{c}\text { Mieles y } \\
\text { Vergara (2020) }\end{array}$ & Constructivista & Cualitativo & $\mathrm{X}$ & $x$ & $x$ & $X$ & & & $X$ \\
\hline \multirow[t]{2}{*}{ Gomera (2008) } & Investigativo & Cualitativo & & & $\mathrm{X}$ & & & & $x$ \\
\hline & & & 3 & 1 & 3 & 2 & 0 & 0 & 4 \\
\hline
\end{tabular}

Elaborado por: Elaboración propia

En la Tabla 4, se señala que tres de los cuatro investigadores han empleado el método investigativo cualitativo y uno de ellos adjuntan a su método el enfoque cuantitativo, mientras que un investigador ha utilizado plenamente el método descriptivo, valiéndose de técnicas óptimas para la recolección de la información; de los cuatro autores ninguno cuenta con sustento teórico en la declaración del método.

\section{DISCUSIÓN}

La pedagogía verde impulsa a amar la Tierra, a ser responsables de las acciones que atentan contra ella y ser reflexivos para encontrar métodos que permitan restablecerla a fin de lograr un desarrollo sostenible medioambiental; es fundamental intervenir en la educación e influir de manera consiente en el pensamiento y actuar de los estudiantes y de la sociedad.

Las alternativas que se han considerado adecuadas para el desarrollo de la pedagogía verde y formación del pensamiento crítico-reflexivo en el campo educativo son: a) Huertos escolares, b) Metodología TiNi, c) Educación ambiental, y d) Desarrollo ecológico. 
Actualmente se atraviesa la era de la ciencia y tecnología, cubiertos de plástico, instrumentos superficiales y realidad virtual, lo que conduce a un apartado natural. Los huertos escolares devuelven el sentir a las actividades agrícolas primordiales para la vida y cultura de la sociedad, integra las diversas áreas curriculares, facilita el desarrollo de competencias y consolida ejes transversales; estos generan experiencias reales entre los estudiantes y la naturaleza con recursos palpables que permite construir aprendizajes duraderos aplicables a la vida cotidiana.

Desde el 2017 el Ministerio de Educación, reconoce a la Metodología Tini como recurso pedagógico de Educación Ambiental y buena práctica en las Instituciones Educativas del Ecuador; los conocimientos, las habilidades y los valores permitirá afrontar la problemática ambiental que se atraviesa, por medio de la transversalización del enfoque medioambiental e integración al sistema educativo, esta metodología orienta una cultura y conciencia responsable hacia el medio ambiente; como una cultura proactiva entre los estudiantes y el medio ambiente, para lograr el desarrollo de una base sólida para la sostenibilidad.

La educación ambiental tiene mucho que contribuir a la construcción del pensamiento crítico-reflexivo, pues enfatiza la preservación y cuidado del medio ambiente, es necesario entender y conocer la problemática ambiental tanto como la reseña socio histórico para analizar la situación y buscar soluciones para contrastarlos. Es primordial el compromiso y dedicación por parte de los docentes, ya que son los encargados de incorporar y encaminar de manera intencional las dimensiones cognitiva, afectiva, conativa de los estudiantes

El desarrollo ecológico posee enfoques que enriquecen los ejes del currículum, manteniendo una estrecha relación entre el ser humano y la naturaleza para desarrollar una conciencia medioambiental formando seres críticos y reflexivos para subsanar el planeta Tierra.

Las alternativas propuestas constituyen un marco de referencia y reflexión para transformar acciones de la sociedad y contenidos de la educación, propiciando el desarrollo de conciencia ambiental como efecto de la reflexión, de la recreación del sí mismo y del mundo empírico del ser humano.

Cabe concluir que para la aplicación de la pedagogía verde y sus alternativos no importa el rol que desempeñes puedes ser: padre de familia, docente, estudiante, directivo o miembro de la comunidad; solo es cuestión de actitud y compromiso, los pequeños cambios generan grandes transformaciones dentro de tu barrio, de la comunidad, sociedad y del mundo entero.

Se considera que, para futuras investigaciones sería conveniente realizar estudios acerca del bosque escuela, por medio de análisis cualitativos y de campo en una población especifica y bajo la premisa de un estudio experimental, lo cual generaría un aporte significativo.

\section{REFERENCIAS BIBLIOGRÁFICAS}

Anguera, T. (1986). La investigación cualitativa. Educar, 23-50. https://educar.uab.cat/article/view/v10-anguera/442

Bermúdez, G., \& Longhi, A. (2008). La educación Ambiental y la Ecología como ciencia. Una discusión necesaria para la enseñanza. Revista electrónica de la enseñanza de las Ciencias, 7(2). 275-296.

Botella, A., Hurtado, A., \& Cantó, J. (2014). Las competencias básicas a través del huerto escolar: una propuesta de proyecto de innovación. En J. Maquilón, \& N. 
Orcajada, Investigación e innovación en formación del profesorado (1 ed., págs. 173-195). Murcia, España:

Cabrera, S. (2014). Beneficios Educativos de los Huertos Escolares. Universidad de la Sevilla, Sevilla.

Castillo-Bustos, M. R. Técnicas e instrumentos para recoger datos del hecho social educativo. Retos de la Ciencia. 5(10), pp. 50-61. https://doi.org/10.53877/rc.5.10.20210101.05

Díaz, T., \& alemán, P. (23 de febrero de 2008). La educación como factor de desarrollo. Universidad Católica del Norte, 1-15.

El Productor. (2017 de diciembre de 2017). Tini, una metodología para la educación ambiental que Ecuador asume. El Productor.

Freire, H. (2011). Educar en verde. Ideas para acercar a niños y niñas a la naturaleza (Vol. 30). Barcelona, España: GRAO.

Freire, H. (2018). Descubre la Pedagogía Verde. https://www.heikefreire.com/empieza-aqui

Freire, H. (20 de Febreo de 2020). Educar no es inculcar - Sobre Educación Ambiental y Pedagogía Verde. Educar no es inculcar - Sobre Educación Ambiental y Pedagogía Verde: https://www.heikefreire.com/2020/02/educar-no-esinculcar-educacion-ambiental-pedagogia-verde.html

Fresneda, C. (septiembre de 28 de 2018). El Mundo. Heike Freire, la educadora 'verde': "El eje de todas las asignaturas debe ser el medio ambiente": https://www.elmundo.es/ciencia-ysalud/ciencia/2018/09/28/5bad1b0aca4741c7728b45ed.html

García, B. (2003). Los huertos escolares: una buena herramienta pedagógica en la educación primaria. Universidad de Valladolid. https://uvadoc.uva.es/bitstream/handle/10324/4606/TFG-

L333.pdf? sequence $=1$ \& isAllowed $=y$

Gomera, A. (noviembre de 2008). La conciencia ambiental como herramienta para la educación ambiental; conclusiones y reflexiones de un estudio en el ámbito universitario. Centro Nacional de Educación Ambiental. https://saneambiente.co/wp-content/uploads/2016/05/01/articulo-concienciaambiental.pdf

Gómez-Luna, E., Fernando-Navas, D., Aponte-Mayor, D., \& Betancourth-Bitriago, L. (abril de 2014). Metodología para la revisión bibliográfica y la gestión de información de temas científicos, a través de su estructuración y sistematización. DYNA, 81(184), 158-163.

Gonzáles, A. (octubre de 2002). La preocupación por la calidad del medio ambiente. Un modelo cognitivo sobre la conducta ecológica. Universidad Complutense de Madrid. https://eprints.ucm.es/id/eprint/4390/1/T26479.pdf

Izaguirre, M. (20 de enero de 2014). La naturaleza en la educación Integral. Universidad Internacional de la Rioja. https://reunir.unir.net/bitstream/handle/123456789/2255/IzaguirreRoitegi.pdf?sequence $=1$ \&isAllowed $=y$

Leguía, J., \& Paredes, N. (2016). Guía introductoria a la metodología TiNi. Ministerio de Educación del Ecuador. https://educacion.gob.ec/wpcontent/uploads/downloads/2017/11/metodo-TINI.pdf

Limón, D., Ruiz-Morales, J. y García L. (2002). Ecociudadanía: Participar para construir una sociedad sustentable. Sevilla: IP

Medina, M. (29 de mayo de 2020). Huerto escolar: características, objetivos, tipos y beneficios. Lifeder. https://www.lifeder.com/huerto-escolar/ 
Mieles, P., \& Vergara, K. (17 de septiembre de 2020). La metodologia TiNi, una práctica para el logro de los objetivos para el desarrollo sostenible 2030. Mamakuna, 1-12.

Novo, M. (30 de marzo de 2009). La educación ambiental, una genuina educación para el desarrollo sostenible. http://www.educacionyfp.gob.es/dam/jcr:8998f1e4-65d7-40dd-94697945013994e8/re200909-pdf.pdf

OMS. (2012). Calidad del aire. Organización panamericana de Salud. https://www.paho.org/es/temas/calidad-aire

Pasek, E. (24 de enero de 2004). Hacia una conciencia ambiental. Redalyc, 8(24), 3414.

Patton, M. (1980). Qualitative evaluation methods. Beverly Hills: Sage.

Pérez de Villarreal, M., \& González, F. (2016). Teaching experimental sciences using Cmap tools. Proc. Of the 7th Int. Conference on Concept mapping, 183-198.

Pérez, M., \& Belletich, O. (marzo de 2018). Innovación metodológica mediante la aplicación de pedagogía verde en Ciencias de la Naturaleza. Universidad Pública de Navarra, 717-733. Navarra, España. 